\title{
Dynamic Pump-Probe Studies of TE and TM Modes in Semiconductor Optical Amplifiers
}

\author{
S. Philippe ${ }^{1}$, F. Surre ${ }^{1,2}$, A.L. Bradley ${ }^{1}$, P. Landais $^{2}$, M. Martinez-Rosas ${ }^{3}$ \\ 1) Trinity College Dublin, Ireland \\ 2) Dublin City University, Ireland \\ 3) UABC, Ensenada, Mexico
}

\begin{abstract}
A contra-propagation set up is implemented and dynamic pump probe studies of a InGaAsP/InP SOA in the gain regime are undertaken using pulses of $2 \mathrm{ps}$ duration. The time resolved amplified probe signal is measured separately for the TE and TM modes of the semiconductor optical amplifier. Different behaviours are observed both in the gain compression and the timescales of the effect, with the TM mode displaying a faster component and a higher gain compression.
\end{abstract}

Keywords: semiconductor optical amplifier, optical switching, gain, polarisation, pump-probe.

\section{INTRODUCTION}

Semiconductor optical amplifiers (SOAs) are mainly used for optical regeneration in optical communication networks. Their main advantage over fibre amplifiers is their potential for integration in all-optical systems. As they exhibit strong non-linearities, they are not only suitable for amplification, but also for signal processing applications. Various configurations have been implemented for all-optical wavelength conversion, based on cross-gain and crossphase modulation $^{1-5}$.

Most of the applications proposed use at least partially fiberised set-ups, where the polarisation state of the light is random or modified as it travels through the fibres. This makes it very difficult both to set the polarisation to a known state and to measure how it is modified. But it is well known that SOAs are polarisation sensitive, with different gain and refractive indices in the TE and TM modes. Although considerable efforts have been made to reduce this sensitivity, using strain and different waveguide structures, and gain anisotropy of less than $2 \mathrm{~dB}$ has been achieved, this does not imply preservation of the state of polarisation of the input beam.

In a wavelength conversion system, a high intensity laser beam (pump) modifies properties (transmission, polarisation and phase) of a low intensity laser beam (probe). Pump-probe techniques can be used to investigate the timescales over which the probe is modified by the pump, by monitoring the probe output as a function of time delay between the pump and the probe pulses. The resolution of such experiments is mainly determined by the width of the pulses. For optical telecommunications applications of polarization rotation effects it is interesting to study the dynamics on the effect in the picosecond regime. In a conventional pump-probe set-up, the pump and probe pulses travel collinearly and in the same direction through the SOA and one is delayed with respect to the other. When the pump and probe are at the same wavelength the only way to separate them is via the polarisation, the most common method being to launch cross-polarised pump and probe pulses and to separate them at the output with a polariser. In the context of a polarisation study this would obviously limit the measurements that could be undertaken. Not only do the pump and probe have to be cross-polarised but their state of polarisation must be preserved as they travel through the SOA. Practically this means they have to be polarised along the eigen modes of the device, leaving only two possible combinations, probe TE pump TM and probe TM pump TE. Although a more complicated heterodyne detection ${ }^{6}$ as been proposed, a simpler and more flexible method to study the polarisation dependence of pump-probe measurements is to use a counter-propagation configuration where the pump and probe pulses travel in opposite directions. Then the beams can simply be separated using a beamsplitter, whatever their polarisation may be.

We propose to use a contra-propagation configuration to investigate if modifications applied to the waveguide in order to achieve polarisation insensitivity can influence the dynamic behaviour of the SOA. In this paper we will present a pump-probe study of dynamic polarisation effects of SOAs in contra-propagation geometry, using picosecond 
timescale pulses. The response for co-polarised pump and probe is measured as a function of pump power for the two eigen-modes of the device and compared in terms of gain compression and timescales, with a view to apply our knowledge to switching applications.

\section{EXPERIMENT}

\section{A. Experimental set-up}

A free space contra-propagation configuration is used, allowing total control and preservation of the state of polarisation of the injected and collected signals, as shown on figure 1 . The $1580 \mathrm{~nm}$ pulsed input is produced using an optical parametric oscillator (OPO), with a repetition rate of $82 \mathrm{MHz}$. The pulse width is $2 \mathrm{ps}$ and the spectral width $4 \mathrm{~nm}$. The pulses are coherent and one is delayed with respect to the other using a variable delay stage. The polarisation of each beam is controlled independently with a quarter wave plate and a half wave plate. Two beamsplitters allow us to monitor the input and output of each beam. The light is coupled in and out of the SOA by two anti reflection-coated achromatic lenses mounted on micro-control 3D translation stages. The device under test is a commercially available $1.5 \mathrm{~mm}$ long bulk InGaAsP/InP travelling wave SOA and is temperature regulated at $20^{\circ} \mathrm{C}$ by means of a Peltier cooler.

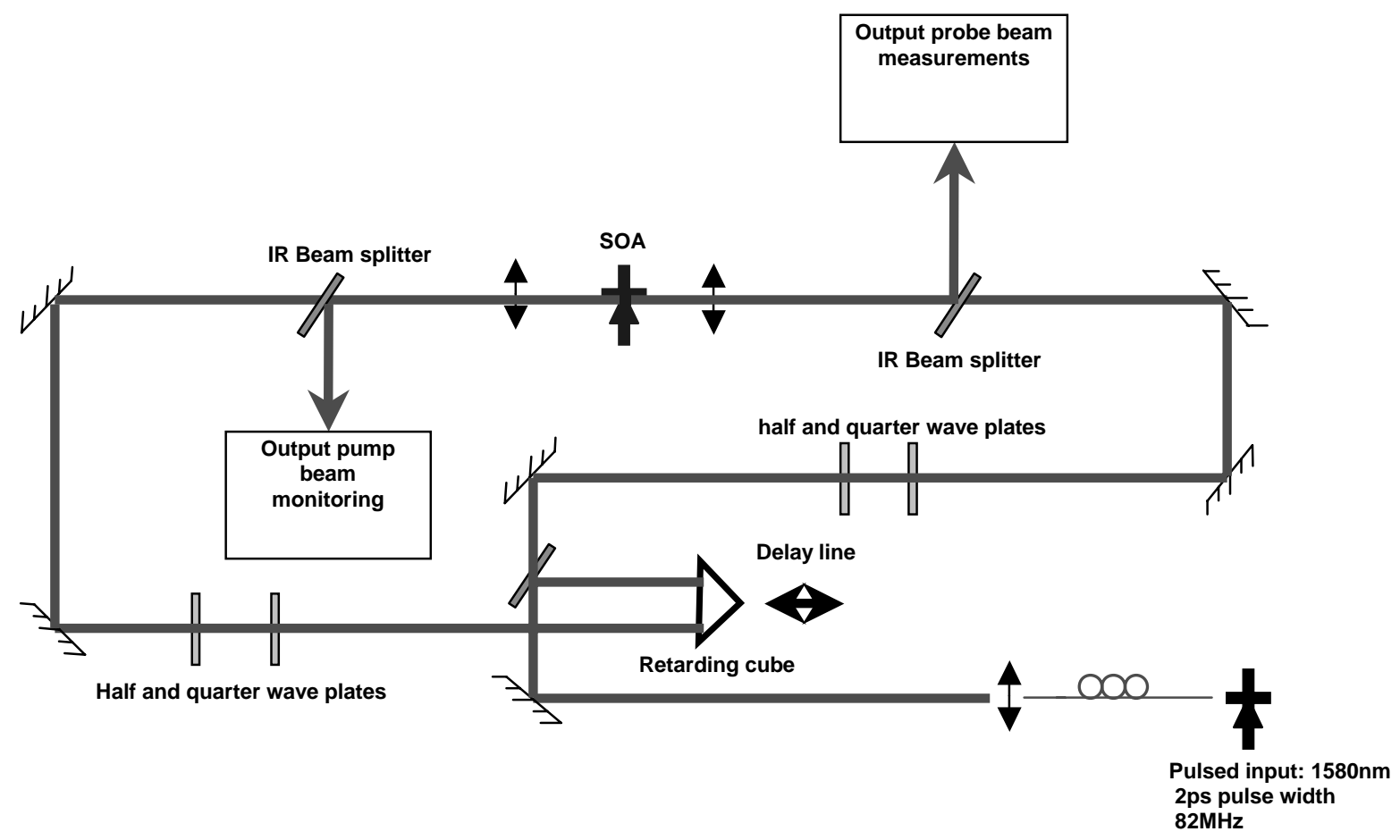

Fig.1. Experimental set-up.

An interference filter is used to isolate the probe signal from the broad ASE at $1580 \mathrm{~nm}$ and the signal is detected using a lock-in technique.

\section{B. Contra-propagation}

As most of previous pump-probe studies of SOAs have been performed in a co-propagation configuration, it is useful to stress the main differences between co and counter propagation. Indeed the transmission curves can look rather similar while the process is different. 
In co-propagation, a negative delay corresponds to the probe travelling ahead of the pump pulse, as the delay increases towards zero, the pump and probe pulses start to overlap and the probe gain decreases. At zero delay they are exactly overlapped. Then as the pump travels further ahead of the probe, the probe is actually sampling the recovery of the device and the probe gain starts to rise again. Thus there is only one value of the delay for which the pulses overlap. In this case, for short length devices, the group velocity mismatch due to different refractive indices in the TE and TM directions is negligible compared to the pulse width ${ }^{7}$ and the pulses stay overlapped over the whole length of the SOA.

In counter-propagation for a range of time delays, the pump and probe pulses overlap at different positions inside the SOA. As a consequence, in this geometry it is also important to note that the displacement of the delay line between the pulses overlapping at the input and them overlapping at output corresponds to twice the length of the SOA.

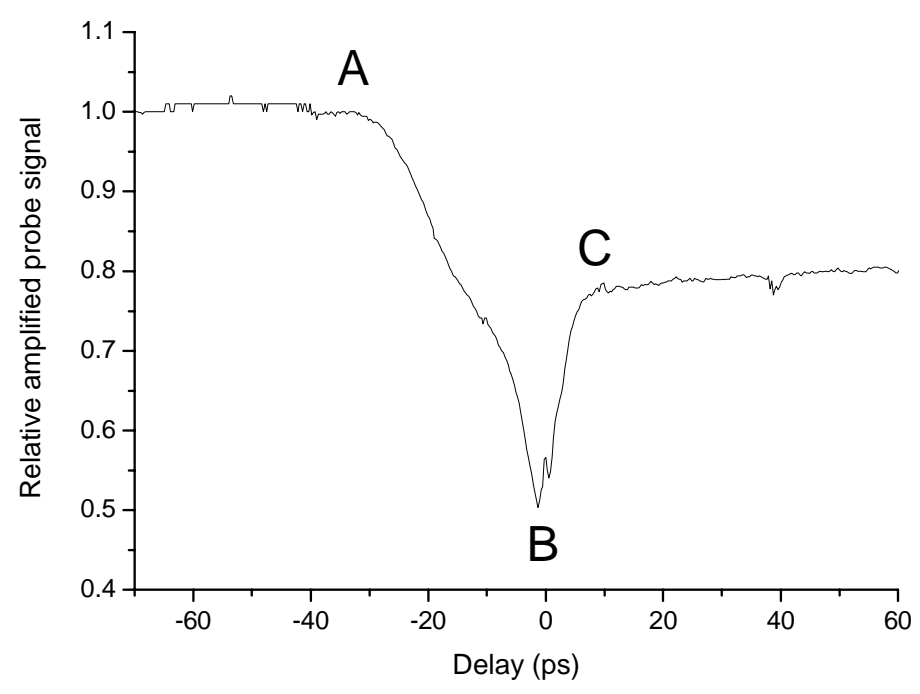

Fig.2 Main features of an amplified probe signal curve (TM mode).

A typical probe signal obtained in counter-propagation is shown in figure 2 . We refer to the probe input facet (pump output facet) as the SOA input and the probe output facet (pump input facet) as the SOA output. Before A, at more negative delays, the probe leaves the SOA before the pump enters it and its transmission is left unchanged. At A, the pump and probe pulses overlap at the output of the SOA. Between A and B the pulses overlap inside the SOA, and as is expected the gain experienced by the probe will be reduced and therefore the intensity of the amplified probe signal will decrease. A minimum is reached at B, where the pulses overlap at the input of the SOA. This minimum can be expected, as it is at this delay that the pump has affected the properties of the entire length of the SOA, with no time for any recovery before the probe arrives. After that point the pump leaves the SOA before the probe enters it and the device has started to recover before the arrival of the probe. As the delay is further increased the amplified probe signal will continue to increase and the recovery time of the device is monitored. The recovery shows two components: a faster recovery component between $\mathrm{B}$ and $\mathrm{C}$, followed by slower recovery due to carrier recombination processes occurring on the timescale of the order of hundreds of picoseconds.

\section{RESULTS AND DISCUSSION}

This paper is concerned with pump-probe experiments performed using co-polarised pulses. From previous experiments with continuous wave lasers ${ }^{8}$, we expect light polarised along the TE or TM direction to travel through the SOA with its polarisation unchanged. To ensure that only light that travels along the TE or TM mode is detected, a polariser is placed in front of the photodiode. The resonant pump and probe wavelength is selected to be $1580 \mathrm{~nm}$, corresponding to the maximum of the SOA gain spectrum. The SOA is biased at $350 \mathrm{~mA}$. The data are taken for a range of pump input powers from $-23 \mathrm{dBm}$ to $-12.2 \mathrm{dBm}$ (corresponding to pulse energies of $61 \mathrm{fJ}$ to $732 \mathrm{fJ}$ ) while the probe input pulse energy is kept constant at $-20 \mathrm{dBm}(122 \mathrm{fJ})$. 
The modification of the amplified probe signal as a function of delay and pump pulse power is presented in figure 3. The output intensity is normalised by the probe signal level that is achieved in the absence of the pump pulse. As the pump energy is increased, the onset of the decrease in the amplified probe signal occurs earlier due to the fact that the depletion of the carriers induced by the higher power pump pulse extends further along the SOA waveguide. The minimum value of the amplified probe signal is also dependent on the pump pulse power. A faster component is also evident in the TM data. The different dynamic components will be discussed below.
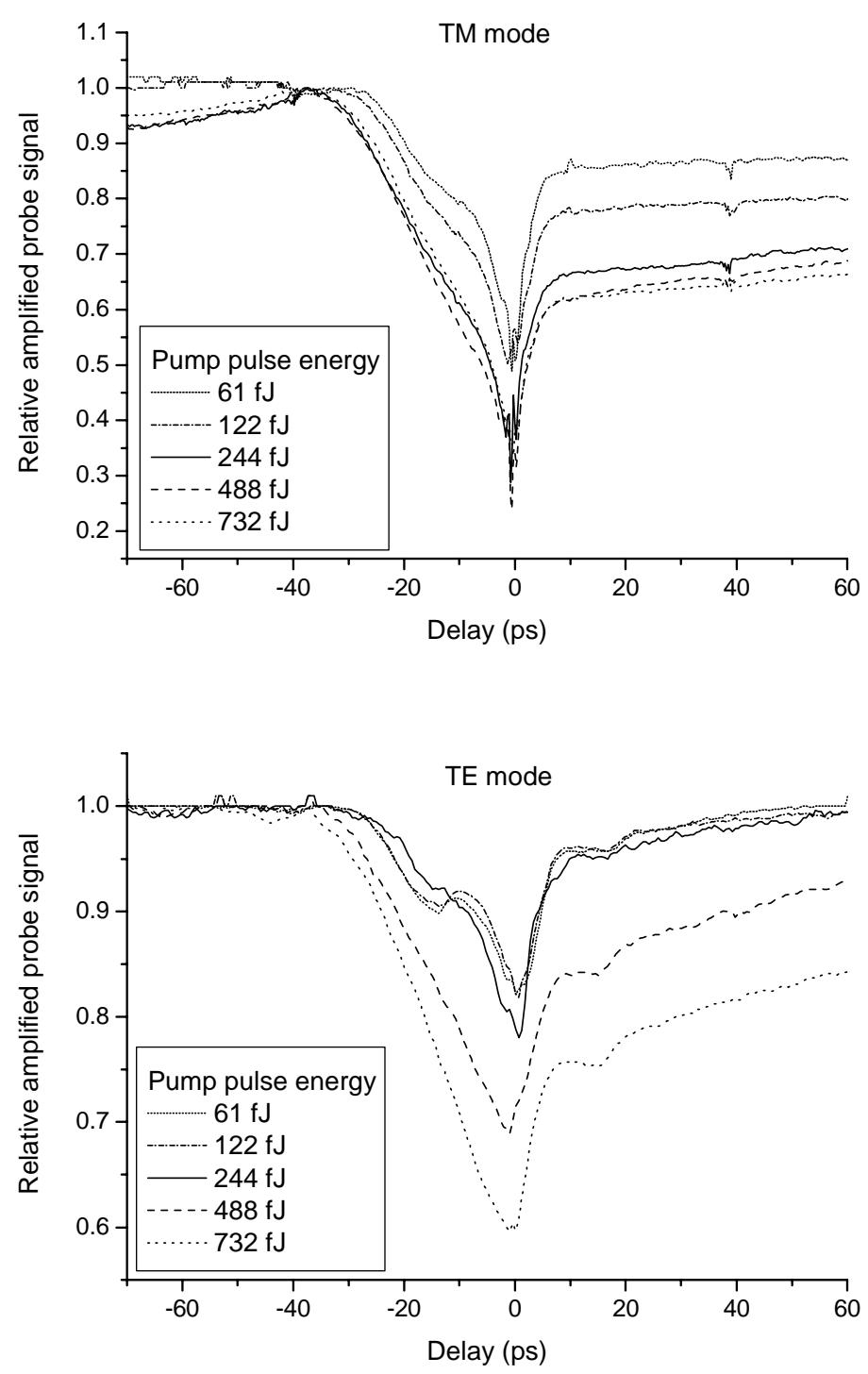

Fig.3 The relative amplified probe signal as a function of delay for a range of pump pulse energies, with constant probe pulse energy of 122fJ. (a) the pump and probe pulse are both launched into the TM mode of the SOA, whereas in (b) they are launched in the TE mode. 


\section{A. Gain compression}

Investigating firstly at zero delay it can be seen that as expected there is gain compression for both polarisations studied. The maximum gain compression at zero delay for each mode in $\mathrm{dB}$ is shown in figure 4 , where the differences in behaviour can be clearly seen. The extent to which the gain of the probe signal is reduced is different in both cases. The compression is always larger in the TM mode with the relative amplified probe signal reduced to between 0.5 and 0.34 , while it does not reach any lower than 0.6 for the TE mode. The compression then stays constant in the TM mode while it continues to increase in the TE mode.

Fig. 4 shows that as the pump pulse energy increases the gain compression increases, as would be expected from carrier depletion effects. The discrepancy between the TM mode gain compression and the TE mode gain compression is most likely due to the strain effects ${ }^{9-11}$. This is commonly present in bulk devices, even for a negligible lattice mismatch ${ }^{9}$, and can also be used in order to equalise the gain in the TE and TM modes ${ }^{12}$. However it also has the consequence of removing the degeneracy of the light and heavy hole valence bands. The carrier density of the light hole band is much lower than that of the heavy hole band and as the TM mode couples preferentially to light holes, we expect gain saturation to happen at lower injected power in TM than in TE.

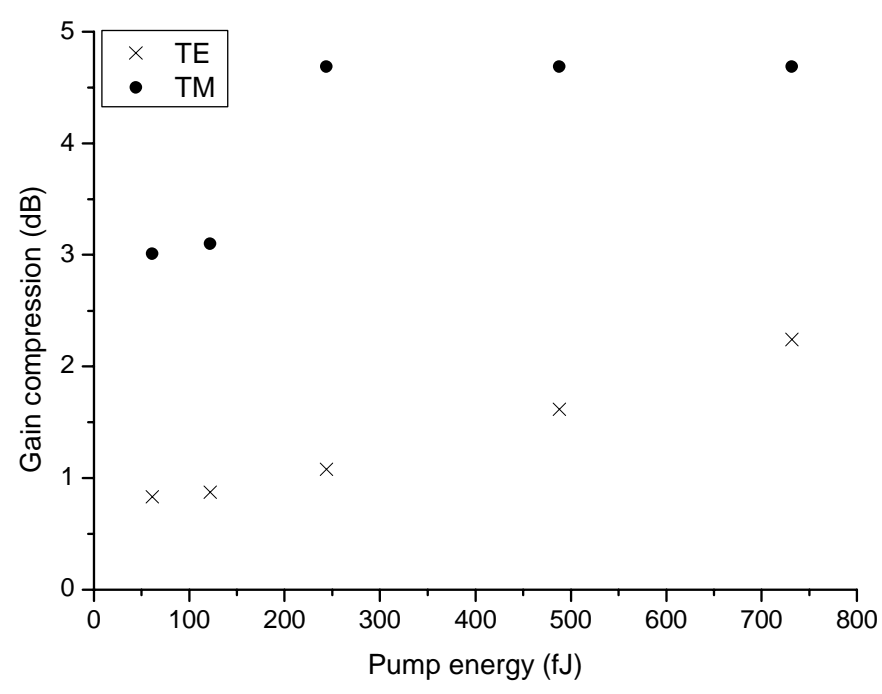

fig.4 Gain compression at zero delay as a function of the pump pulse energy. (Probe pulse energy: 122fJ).

\section{B. Timescales}

In order to compare the timescales, we define the fall time $\left(t_{f}\right)$ as the time for the probe transmission to decrease from $10 \%$ to $90 \%$ of its lowest value and the fast recovery time $\left(t_{r}\right)$ as the time for the probe transmission to increase back from $10 \%$ to $90 \%$ of its value after the first recovery component observed. Those times correspond to the $\mathrm{AB}$ and $\mathrm{BC}$ regions on figure 2 and are shown in figure 5, where the output probe signal is normalised from zero (no effect of the pump) to -1 (maximum gain compression).

The fall time for the TE and TM mode dynamics as a function of the input pump energy is shown in figures 6 . This corresponds to the pulses overlapping inside the SOA and stays constant at about $25 \mathrm{ps}$ for the TE mode even though the decrease in the amplified probe signal starts earlier. In fact in the TE mode the slope of the $\mathrm{F}_{1} \mathrm{~F}_{2}$ section stays constant. This does not happen for the TM mode. Due to the sharper feature appearing in the valley, $\mathrm{F}_{2}$ always occurs at the same delay and thus when $F_{1}$ happens at lower delays, as the pump power gets higher, the fall time increases from $21 \mathrm{ps}$ to $29 \mathrm{ps}$.

The fast recovery time (figure 7) on the other hand is constant for the TM mode, around 5ps. It is slightly higher in the TE mode than in the TM mode, and increases from 5ps to 6.7ps with pump energy, except for the highest injected pump power, although more data are needed to confirm this trend. The second part of the recovery, which corresponds to 
slower carrier recombination processes, cannot be measured as precisely, as we are limited by the length of our delay line. However it can be seen that the gain has recovered at 60ps time delay in TE for the lowest pump energy and a maximum time constant of 200ps was measured in TM.

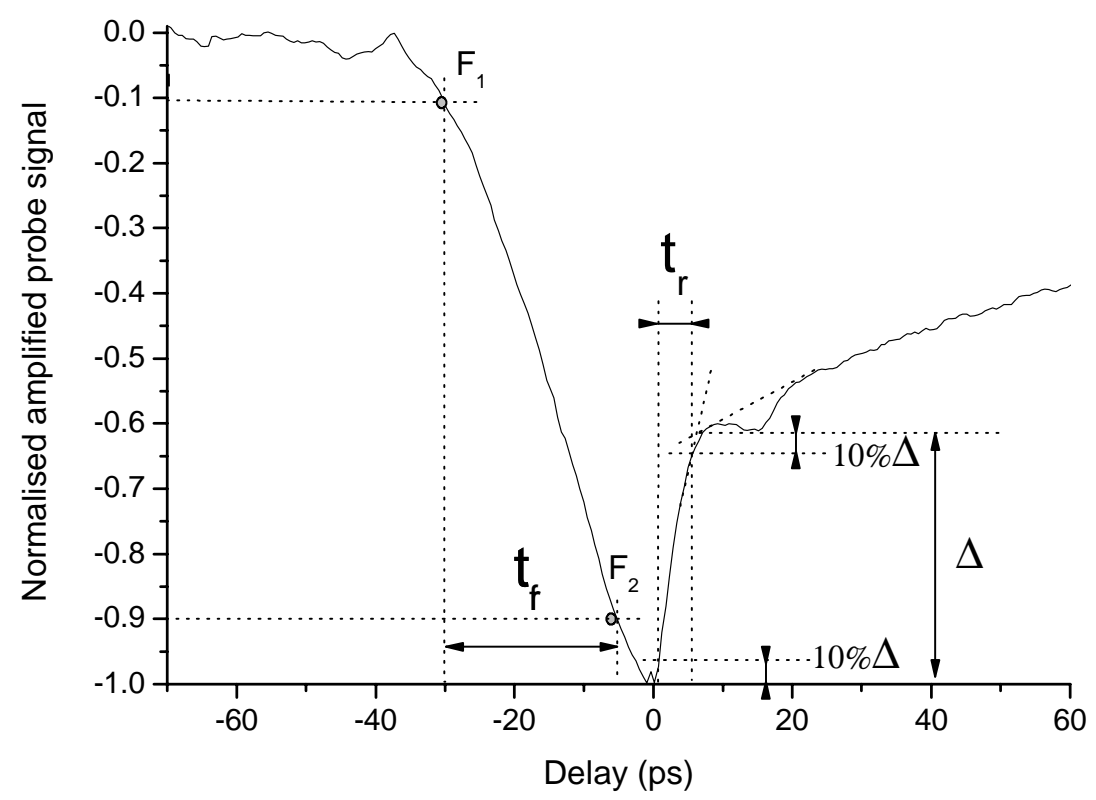

Fig.5 Characteristic times measurements from pump-probe curve (TE mode).

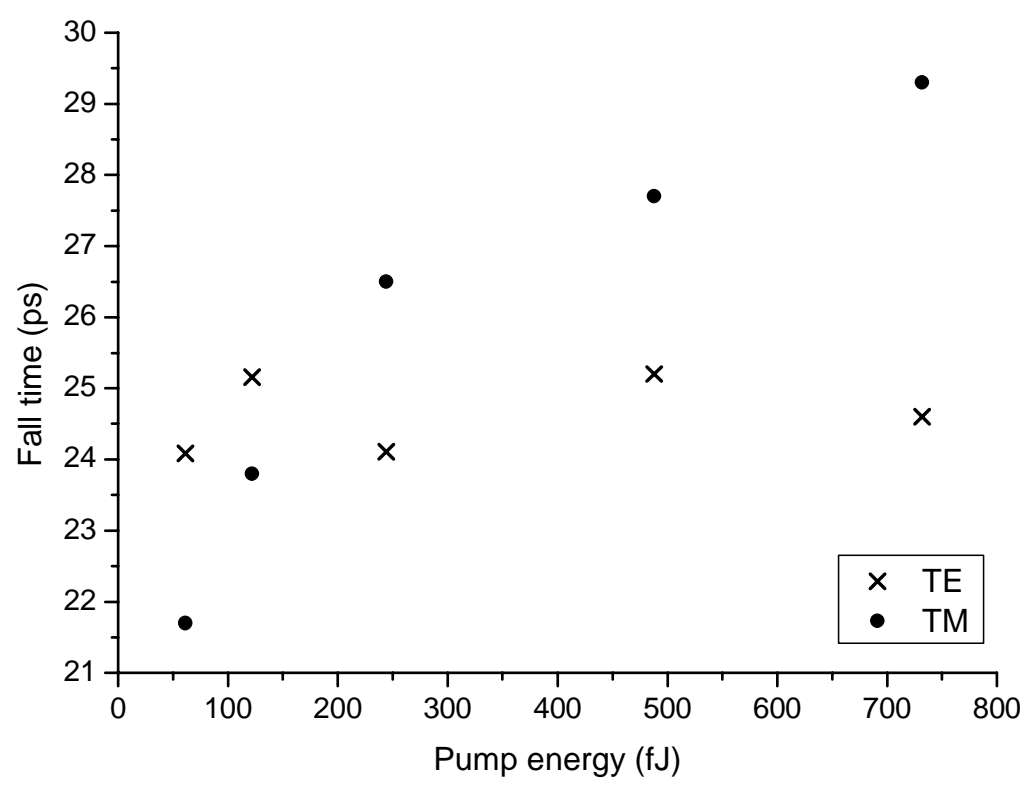

Fig. 6 Fall time of TE and TM modes as a function of pump energy. 


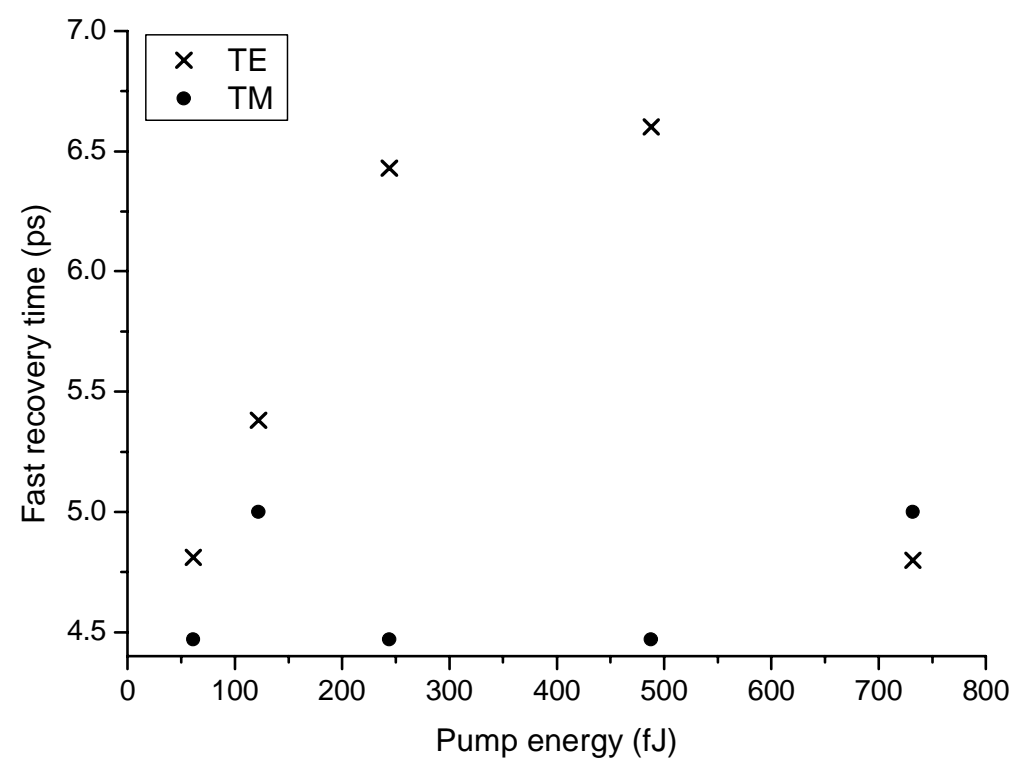

Fig. 7 Fast recovery time of TE and TM modes as a function of pump energy.

Clearly the processes associated with the TE and TM modes near zero time delays are different. One possible explanation is that strain effects are dominating the dynamics in this region. The preferential coupling of the TM mode to transitions involving the light holes ${ }^{12}$ and their faster recombination rate suggests that the faster feature observed in TM could be associated to the light hole dynamics. This has been observed before ${ }^{13}$ in co-propagation in a quantum well SOA using a heterodyne pump-probe technique with $130 \mathrm{fs}$ pulses. As mentioned above, a small lattice mismatch is enough to remove the degeneracy of the light and heavy hole valence bands. A strong polarisation dependence of both the gain compression and characteristic times has been observed.

\section{Switching}

In the context of switching applications, higher gain compression and fast recovery times are two features of great interest. In the TM mode the fact that the gain compression is independent of pump power at higher pump energies is also an advantage. The potential for switching applications is not ruled out by the presence of the slower carrier recombination component. Other groups have suggested two different methods to overcome this. The first is simply not to allow the device to recover completely between the data pulses. Only the fastest effects are thus used but the contrast ratio is decreased ${ }^{14}$. The second option involves CW pumping at transparency in order to speed up the slower effects ${ }^{15}$.

\section{CONCLUSION}

We have shown that a contra-propagating free-space experimental bench allows for comprehensive investigation of the polarisation dependent dynamics in SOA devices, including but not limited to co-polarised pump and probe pulses. In this paper we have presented an investigation of the SOA dynamics in the TE and TM mode with resonant picosecond pulses. Under the same experimental conditions the dynamics in the TE and TM modes were found to be different. The gain compression is higher for the TM mode and the fall time increases with pump power from $21 \mathrm{ps}$ to $29 \mathrm{ps}$. This is not the case in TE where the fall time stays constant at $25 \mathrm{ps}$. There is a faster timescale component in the recovery of the amplified probe signal in the TM mode. Clearly the dynamic behaviour of the SOA is polarisation sensitive, even though this device is commercially sold as polarisation insensitive. This is probably due to the modification of the valence band structure by strain. 


\section{Acknowlegments}

This work is supported by:

Enterprise Ireland under the Proof of Concept Grant PC/2004/331

The Irish Research Council for Science, Engineering and Technology

\section{REFERENCES}

[1]Asghari, M., White, I.H., and Penty, R.V.: 'Wavelength conversion using semiconductor optical amplifiers.', IEEE Journal of Lightwave Tech., 1997, 15, (7), pp.1181-1190.

[2]Soto, H., Erasme, D., and Guekos, G.: 'Cross polarization modulation in semiconductor optical amplifiers,' IEEE Photon. Technol. Lett., 1999, 11, pp. 970-972.

[3]Patrick, D.M., Ellis, A.D., Davies, D.A.O., Tatham, M.C., and Sherlock, G., 'Demultiplexing using polarization rotation in a semiconductor laser amplifier,' Electronic Letters, 1994, 30, (4), pp 341-342.

[4]Liu, Y., Hill, M.T., Tangdiongga, E., de Waardt, H., Calabretta, N., Khoe, G.D., and Dorren, H.J.S.: 'Wavelength conversion using nonlinear polarization rotation in a single semiconductor optical amplifier', IEEE Photonics Tech. Letters, 2003, 15, (1), pp 90-92.

[5]Stephens, M.F.C., Asghari, M., Penty, R.V., and White, I.H.: 'Demonstration of ultrafast all-optcal wavelength conversion utilizing birefringence in semiconductor optical amplifiers', IEEE Photonics Tech. Letters, 1997, 9, (4), pp 449-451.

[6]Borri, P., Langbein, W., Mork, J., Hvam, J.M., 'Heterodyne pump-probe and four-wave mixing in semiconductor optical amplifiers using balanced lock-in detection', Optics Communications 169 (1999) 317-324.

[7]Tang, J.M., Spencer, P.S., Rees, P., Shore, K.A., 'Pump Power Dependence of Transparency Characteristics in Semiconductor Optical Amplifiers' IEEE J. Quant. Elec., vol.36, no.12, December 2000.

[8] Kennedy, B.F., Philippe, S., Landais, P., Bradley, A.L., Soto, H., 'Experimental investigation of polarisation rotation in semiconductor optical amplifiers', IEE Proceedings - Optoelectronics, Vol. 151, No.2, April 2004, pp114-118.

[9]Wang, W., Alaart, K., Lenstra, D., 'Gain Anisotropy in Semiconductor Optical Amplifier: Confinement Factors or Material Gain', IEEE ICTON 2004, pp314-317.

[10]Visser, T.D., Demeulenaere, B., Haes, J., Lenstra,D., Baets, R., Blok, H., 'Confinement and Modal Gain in Dielectric Waveguides', J. Lightwave. Tech., vol.14, no.5, may 1996.

[11]Visser, T.D., Blok, H., Demeulenaere, B., Lenstra,D., 'Confinement Factors and Gain in Optical Amplifiers', IEEE J. Quant. Elec., vol.33, no.10, October 1997.

[12]O'Reilly, E.P., Adams, A.R., 'Band-Structure Engineering in Strained Semiconductor Lasers', IEEE J. Quant. Elec., vol.30, no.2, February 1994.

[13]Lenz, G., Ippen, E.P., Wiesenfeld, J.M, Newkirk, M.A., Koren,U., 'Femtosecond dynamics of the nonlinear anisotropy in polarization insensitive semiconductor optical amplifiers', Appl. Phys. Lett. 68 (21), 20 May 1996.

[14]Uskov, A.V., O’Reilly, E.P., Manning, R.J., Webb, R.P., Cotter, D., Laemmlin, M, Ledentsov, N.N, Bimberg, D., 'Prospects for ultrafast optical switching based on quantum dot semiconductor optical amplifiers in nonlinear interferometers.' CLEO 2004, vol.2, 16-21 may 2004.

[15]Dupertuis, M.A., Pleumeekers, J.L., Hessler, T.P., Selbmann, P.E., Deveaud, B., Dagens, B., Emery, J.Y., 'Extremely Fast High-Gain and Low-Current SOA by Optical Speed-Up at Transparency', IEEE Photonics Technology Letters, vol.12, no.11, November 2000. 